\title{
Enquêter entre interdictions et non-dits au sein d'un programme de coopération bilatérale au Vietnam
}

Une heuristique des contretemps

\section{Mikaëla Le Meur}

\section{OpenEdition}

Journals

Édition électronique

URL : http://journals.openedition.org/anthropodev/298

DOI : 10.4000/anthropodev.298

ISSN : 2553-1719

Éditeur

APAD - Association pour l'anthropologie du changement social et du développement

Édition imprimée

Date de publication : 1 septembre 2014

Pagination : 47-70

ISBN : 9791093476018

ISSN : 2276-2019

Référence électronique

Mikaëla Le Meur, « Enquêter entre interdictions et non-dits au sein d'un programme de coopération bilatérale au Vietnam », Anthropologie \& développement [En ligne], 40-41 | 2014, mis en ligne le 01 septembre 2016, consulté le 20 avril 2019. URL : http://journals.openedition.org/anthropodev/298 DOI : 10.4000/anthropodev.298

La revue Anthropologie \& développement est mise à disposition selon les termes de la Licence Creative Commons Attribution 4.0 International. 


\title{
Enquêter entre interdictions et non-dits au sein d'un programme de coopération bilatérale au Vietnam
}

\author{
Une heuristique des contretemps
}

Mikaëla Le Meur

Depuis la fin des années 1980 avec le Doi moi (renouveau), le Vietnam s'est ouvert à l'établissement de nombreux projets de développement étrangers. Face à une communication gouvernementale répondant aux attentes des institutions internationales en matière de réformes de la gouvernance, l'étude du fonctionnement quotidien des institutions en charge des politiques publiques paraît nécessaire. Cet article propose un retour réflexif sur une enquête ethnographique réalisée au sein d'un projet de coopération bilatérale dans une province du centre du pays. L'examen des conditions pratiques de l'enquête est destiné à alimenter la réflexion sur le fonctionnement des institutions, depuis l'Etat local vietnamien jusqu'au projet bilatéral provincial. Entre contrôles bureaucratiques et policiers, difficultés d'énonciation des problèmes publics et censure stratégique, l'expérience pratique du chercheur et ses difficultés d'enquête nourrissent la compréhension du contexte d'intervention et l'analyse de la mise en œuvre des politiques publiques au quotidien.

Since the end of the 1980's with the Doi moi (renovation), Vietnam has opened its borders to the establishment of numerous foreign development projects. While the government communicates to satisfy the requirements of international institutions for reforming governance, it seems necessary to investigate the daily functioning of the institutions in charge of public policies implementation. This article presents a reflexive feedback from an ethnographic research carried out within a bilateral cooperation project in a central province of the country. The examination of practical investigation conditions gives insightful information about the operation of institutions, from local Vietnamese State to the provincial bilateral project. Between bureaucratic and police controls, constraints on speech referring to public issues and strategic censorship, the researcher's practical experience and the difficulties he faces eventually help un- 
derstanding the context of intervention and analysing the daily implementation of public policies.

\section{Introduction}

Avant l'ouverture du pays avec le Doi moi (renouveau) adopté en 1989, Dinh Trong Hieu recensait les difficultés d'accès au terrain pour un chercheur en sciences sociales dans la République Socialiste du Vietnam (Dinh Trong Hieu, 1986). Selon lui, la " méfiance idéologique " du gouvernement, craignant la contamination capitaliste, était une des raisons du contrôle exercé sur les activités des chercheurs étrangers. L'" espionite ", liée à I'héritage historique du Vietnam au XXe siècle marqué par la quasi permanence de la guerre (contre le Japon, la France, les États-Unis, le Cambodge, la Chine, etc.), aurait alimenté cette paranoïa gouvernementale. Dinh Trong Hieu relevait par ailleurs le caractère utilitariste et positiviste de la culture scientifique socialiste. L'approche empirique et qualitative des sciences sociales serait alors incompatible avec la manière dont le gouvernement vietnamien décrète le réel (Scott et al., 2006) sur la base de l'idéal révolutionnaire d'une marche moderniste vers le progrès. Le principe même de l'enquête ethnographique poserait donc problème dans ce contexte de contrôle suspicieux doublé d'une incompréhension fondamentale du travail du chercheur.

Depuis les années 1990, le Vietnam a adopté une forme de capitalisme d'Etat et s'est ouvert aux investissements étrangers. Le pays accueille désormais massivement des projets de développement, soutenu dans sa communication par certains chercheurs proches des bailleurs internationaux, vantant la vitalité de la "société civile " vietnamienne (Nørlund, 2007). Du fait de cette évolution majeure, il convient de questionner à nouveau les conditions pratiques d'une enquête ethnographique en contexte vietnamien. Ma réflexion mobilisera des données ethnographiques produites en 2011 lors d'une enquête réalisée au sein d'un programme de développement mené conjointement par une administration provinciale vietnamienne et un organisme de coopération européen.

Destinée à améliorer la gestion des déchets et l'irrigation rurale dans différents districts d'une province du centre du pays, cette institution bilatérale $\mathrm{m}$ 'a accueillie afin que je réalise une enquête indépendante sur la 
question des déchets dans deux localités concernées par ses actions. Si le projet initial était celui-ci, des difficultés m’ont amenée à revoir mon enquête. J'ai alors produit deux types de matériaux empiriques. (1) Le premier, que je qualifie de conventionnel (nous verrons que cela n'est pas synonyme d'officiel, ni d'autorisé), repose sur divers séjours dans deux petites villes concernées par le projet. Il s'agissait d'étudier la question des déchets sous l'angle de la gouvernance urbaine dans son " usage exploratoire et descriptif ${ }^{1}$ des pratiques de gestion d'un problème public urbain (Blundo et Le Meur, 2009 : 8). J'ai réalisé des entretiens avec les usagers de la collecte des ordures, les employés des services de gestion et les fonctionnaires locaux. (2) L'autre matériau empirique a été produit de manière non conventionnelle : la situation d'enquête n'a jamais été explicitée auprès de mes interlocuteurs, les membres du projet installés dans la capitale provinciale. C'est en partageant le quotidien de l'institution, depuis certaines réunions internes, aux activités officielles, en passant par des discussions informelles sur les arcanes du projet, que j'ai pu constituer ce matériau.

La réflexion sur ces deux types d'enquête sera organisée autour de la notion d'information, considérée à la fois comme discours et comme pratique inscrite dans des interactions. Je présenterai les enjeux de pouvoir liés à l'accès, à la circulation ou encore à l'instrumentalisation de contenus informationnels dans mon rapport avec les institutions locales. II ne s'agit pas de décrire des difficultés d'enquête en soi, mais bien de questionner la valeur heuristique de la mise en lumière de tels usages informationnels pour la construction du sens dans la recherche anthropologique. Aussi, la notion d'information sera également utilisée dans le sens d'une " matrice organisationnelle " (Morin, 2005 : 37), c'est-à-dire dans sa capacité à mettre en évidence les logiques d'acteurs, leurs réseaux d'interrelations et les logiques institutionnelles que la socio-anthropologie du développement et des politiques publiques se donne pour but d'étudier.

${ }^{1}$ Ma traduction. 


\section{Négocier l'enquête : aiguillages, blocages et détours}

Dans un premier temps, je proposerai de réfléchir aux différentes contraintes qui s'exercent sur le chercheur dans son accession au terrain et à la manière dont il en négocie les contours avec les différentes institutions auxquelles il est confronté. Les aiguillages, blocages et détournements de la recherche permettront de mettre en évidence des représentations ainsi que des pratiques institutionnelles alimentant la réflexion sur l'objet d'étude initial.

\section{Intercessions, filtrages et lignes tracées}

Si l'époque indochinoise est révolue, la capitale provinciale, siège du projet de développement et première escale de l'anthropologue, pourrait s'apparenter à un "pré-terrain " postcolonial, où le chercheur s'insère dans la réalité locale par un jeu d'assignations identitaires (Condominas, 1965). C'est un lieu de rencontres, où il se confronte à diverses représentations sur le terrain et sur la manière dont il doit l'aborder. C'est aussi, plus concrètement, le lieu où se négocie le partenariat tacite avec l'institution du projet.

Peu d'étrangers " blancs ${ }^{2}$ » résident durablement dans la capitale provinciale (une douzaine maximum). Ce groupe est caractérisé par un certain entre soi, lié à la fréquentation des mêmes immeubles d'habitation "luxueux" aux yeux de beaucoup de Vietnamiens, des mêmes restaurants, des mêmes commerces. La majorité ne parle pas le vietnamien, bien que beaucoup disent avoir essayé au début et renoncé par la suite du fait de la difficulté de la langue ${ }^{3}$ et de leurs obligations professionnelles. Leur réseau de connaissances vietnamiennes (hors recours à un interprète dans le cadre du travail) est donc majoritairement anglophone.

Mes premiers contacts avec des Vietnamiens étaient donc déterminés par ce réseau de connaissances. A l'évocation de mon idée d'enquête, ces

${ }^{2}$ Comptons arbitrairement les expatriés européens, nord-américains, australiens, néozélandais, etc.

${ }^{3}$ Le vietnamien est une langue tonale (6 tons), ce qui rend sa prononciation et sa compréhension difficile. 
interlocuteurs m'ont en général mise en garde contre ce projet délirant de vivre dans les districts (c'est-à-dire à la campagne). Mon professeur de vietnamien, par ailleurs investi dans divers projets de développement (Banque Mondiale, Unicef, Handicap International, etc.), a ainsi dépeint une image négative de la campagne en me recommandant de n'y faire que des allers-retours d'une journée en voiture avec chauffeur, ce que font d'ailleurs tous les expatriés, selon lui. "Mais, es-tu au moins déjà allée à la campagne au Vietnam [...] ? " m'a ainsi demandé une amie des membres du projet, déconcertée, "Parce que c'est très pauvre [...], c'est très rudimentaire... ". J'ai ainsi été assignée au groupe jugé homogène des "blancs", attachés au confort moderne, aux distractions citadines, à la santé fragile, etc.

Au-delà de ces projections identitaires, ce sont aussi des représentations de la campagne nourries par certains citadins vietnamiens qui déterminent ce discours. Le gouvernement socialiste est d'ailleurs marqué par une représentation ambivalente du monde rural. La campagne est d'un côté une source de résistances au changement et subit donc une politique volontariste, notamment avec la révolution verte. Alors qu'en parallèle, le gouvernement glorifie les révoltes "paysannes" contre l'oppresseur étranger ou capitaliste (comme dans le musée de l'une des localités de l'enquête).

Dans ces conditions, il fût difficile de trouver un interprète (mon vietnamien étant balbutiant) acceptant de m'accompagner pour plusieurs jours dans les districts. Ce sont finalement deux étudiantes, jeunes diplômées de l'université d'anglais et issues de milieux modestes, qui m'ont accompagnée tour à tour. La première, troisième enfant d'une grande fratrie de la campagne du centre, a été d'une grande aide pour " débloquer » l'enquête.

Outre l'influence de ces représentations croisées sur ce que devait être la recherche de terrain, le caractère ambigu de mon accord avec le projet de développement a entraîné une négociation permanente de mon enquête. Mon objectif initial était essentiellement d'être soutenue (notamment auprès de l'administration vietnamienne), en échange d'une étude sur la gestion des déchets qui intéresserait les membres du projet. Durant ma première entrevue avec le directeur européen, qui $m$ 'avait d'emblée déclarée "freelance " et sans obligations à tenir vis-à-vis du projet, je lui ai 
demandé son avis pour choisir deux communes, dans les districts concernés par leurs actions :

"Le district 1 semble tranquille et proche de la capitale. [...] Le district 4 est une très mauvaise idée parce qu'il y a des problèmes. Mais ce ne sont pas des problèmes intéressants par rapport aux déchets, ce sont des problèmes liés au développement en général. Ils profitent de la présence de projets étrangers. [...] Ils savent communiquer et recevoir les experts, mais dans les faits ils ne font rien d'intéressant. " (Carnet de terrain, 30.08.2011)

Malgré ma curiosité pour le district 4, où des enjeux liés à la captation de l'aide au développement semblaient se dessiner, j'ai suivi la voie proposée. Si le district 1 est tranquille, outre son environnement bucolique, c'est que les partenaires locaux sont jugés coopérants avec le projet. Aussi, après quelques temps, j'ai choisi une seconde localité où certains problèmes liés à la politique du projet pouvaient émerger, notamment un conflit lié à la localisation d'un site de décharge d'ordures dont j'avais eu vent. Cette ville du district 2 faisait face à des enjeux proches de la première (taille équivalente, accession à un statut urbain supérieur en cours), aussi j'ai proposé au directeur une comparaison, qu'il accepta. Cependant il me déconseilla de $m^{\prime}$ intéresser au site de décharge. L'intérêt pour lui résidait dans les pratiques quotidiennes des citadins avec leurs déchets, un sujet de recherche a priori déconnecté des enjeux politiques locaux dans lesquels le projet était pris ${ }^{4}$.

Si l'indépendance est un leurre vite écarté, le compromis entre les deux parties et les concessions dans la recherche déterminent au contraire sa pérennisation. Aussi les membres du projet, se représentant les localités partenaires et imaginant les bénéfices qu'ils peuvent tirer d'une étude de leur "terrain ", influencent-ils inévitablement l'enquête. Celle-ci est par ailleurs alimentée par ces nouvelles informations, qui permettent aussi au chercheur de s'orienter. Par la suite, il a été très fructueux d'analyser le décalage entre mes observations dans ces deux districts et les représenta-

\footnotetext{
${ }^{4}$ Le directeur tentera souvent, plus ou moins explicitement, de me faire abandonner les enjeux institutionnels de la gestion des déchets, pour m'amener à me concentrer sur les pratiques des habitants.
} 
tions des membres du projet sur leurs "facilités " ou leurs " difficultés » de coopération.

\section{Parcours institutionnels et réseaux d'interlocuteurs}

Le gouvernement vietnamien exige des chercheurs qu'ils possèdent des autorisations d'enquête délivrées par les autorités administratives compétentes. Certains témoignent avoir modifié leur objet de recherche dans leur quête délicate pour obtenir les " tampons rouges " (Scott et al., 2006 : 31 ; Turner et al. , 2011). Ils soulignent également la difficulté à tenir leur objectif d'empirisme quand les procédures pour organiser des rencontres sont si lourdes et que les contrôles administratifs sont si pressants (Bonnin, 2011). Ma recherche n'a pas échappé à ces règles. Mais tous ces contacts parfois embarrassants avec l'administration doivent être considérés comme des moments privilégiés d'appréhension du fonctionnement de l'Etat local au quotidien.

Un des principaux obstacles de mon accès à l'information réside dans mes difficultés à obtenir une lettre de recommandation, document nécessaire pour effectuer une recherche en règle. Elle devait m'être délivrée par l'administration provinciale associée à la coopération européenne, représentée par le vice-directeur vietnamien de la cellule de pilotage du projet. A chaque pas en avant, la procédure d'obtention se complexifiait car le vice-directeur déclarait certains documents non conformes, demandait de nouveaux justificatifs et de nouvelles précisions sur mon projet. Les autorités vietnamiennes ont parallèlement lancé une enquête, apparemment d'usage pour tout étranger prétendant travailler au Vietnam, sur ma personne. Sans la lettre, les fonctionnaires du projet m'ont d'abord expliqué que je $n^{\prime}$ avais pas le droit de me rendre dans les districts ${ }^{5}$. Faute de temps, j'ai décidé de commencer l'enquête sans ce document ${ }^{6}$, sillonnant les communes à la rencontre des habitants et cherchant à obtenir quelques rendez-vous avec des petits fonctionnaires.

\footnotetext{
${ }^{5}$ Avec un visa touristique, $n^{\prime}$ importe quel étranger a le droit de sillonner le territoire vietnamien. Beaucoup de chercheurs travaillent d'ailleurs avec un visa de ce type, parfois accompagné d'autorisations officielles, tant les visas de recherche sont complexes à obtenir (et ne résolvent pas tous les problèmes d'accès au terrain).

${ }^{6}$ Qui ne m'a finalement été délivré que trois semaines avant mon départ.
} 
Dans la commune du district 2, les débuts de l'enquête ont été particulièrement laborieux. Dès l'enregistrement auprès des autorités de police (procédure obligatoire pour tout étranger), les officiers voulaient que je parte de la commune, avant finalement de m'autoriser à séjourner à I'hôtel. C'est en négociant âprement que mon interprète et moi avons eu l'autorisation de rester chez l'habitant. Notre deuxième séjour nous permit de découvrir qu'il ne s'agissait que d'un répit temporaire car notre logeuse initiale refusa tout contact avec nous, arguant que la police était venue la questionner chez elle après notre départ. Après avoir trouvé une nouvelle hôtesse, nous sommes allées déclarer notre présence à la police. La police nous offrit une seule nuit de répit avant de venir enquêter chez notre nouvelle logeuse en notre absence. De retour d'une journée de travail, elle nous somma de quitter sa maison, inquiète d'être mêlée à nos activités et effrayée par le policier venu l'interroger: "j'ai pensé qu'il s'agissait d'un crime ! ". Il est difficile de définir l'événement comme un contrôle de routine ou un traitement spécial réservé à un enquêteur non désiré. La règle est d'ailleurs elle-même sujette à interprétations : certains Vietnamiens sont persuadés qu'héberger des étrangers est formellement interdit, quand d'autres les accueillent volontiers. Tant que la police locale est mise au courant et qu'elle tolère la pratique, il ne semble pas y avoir de problème. Cependant, dans un cas d'asymétrie dans l'information sur le droit en vigueur et de variations dans l'application de la règle, contrôles et intimidations policières risquent d'être plus fréquents et de mettre un sérieux frein à la recherche.

Si l'institution bureaucratique dans son ensemble peut être vue comme un obstacle, notamment lorsqu'elle se double de contrôles policiers, dans le quotidien de l'enquête l'ethnographe interagit avec des personnes situées au sein d'un système réticulaire régi par des mécanismes de fermeture et d'ouverture.

Des gatekeepers " directement ou indirectement, facilitent ou entravent l'accès des chercheurs à des ressources telles que des personnes, des institutions, des informations et des éléments de logistique. 》(Bonnin, 2011 : 12)

Dans ce réseau constitué, l'ethnographe navigue à vue et, de proche en proche, se voit faciliter des rencontres ou, au contraire, se "ferme " une partie du terrain. 
Si certaines personnes ont fait rempart à mon enquête, comme le vicedirecteur du projet faisant des difficultés pour me délivrer l'autorisation de recherche, d'autres l'ont soudainement facilitée. Notre dernière logeuse dans la seconde commune fait partie de ces gateopeners providentiels, dans un contexte de contrôle policier très marqué. C'est à l'occasion de multiples visites au Comité Populaire de la commune que nous avons rencontré cette jeune fonctionnaire en charge de questions agricoles. En sympathisant, elle nous a proposé de nous loger. Du fait des multiples déboires avec nos précédentes hôtesses, je craignais que la police ne vienne l'intimider. Elle m'assura qu'il n'y avait rien à craindre car elle connaissait les fonctionnaires de police du commissariat communal. Etant elle-même fonctionnaire communale, de père ancien fonctionnaire communal et désormais représentant de quartier, elle semblait jouir des relations qui nous manquaient jusque-là.

A l'inverse, l'enquête dans la première commune, qui se déroulait sans encombre depuis le début, s'est soudainement "refermée ". A chaque visite au commissariat pour m'enregistrer, je n'ai eu aucun problème sauf lors de mon dernier séjour, où j'ai été convoquée par un officier supérieur :

Le policier gradé nous explique que des gens sont venus se plaindre à lui de mes interviews. On lui a dit que les questions que je pose relèvent $d$ " "affaires nationales " et que mes interlocuteurs se sont sentis en danger de répondre. "[...] Je devrais m'en tenir à des questions strictement liées aux déchets et à l'environnement et ne pas poser de questions sur la population des zones résidentielles. Ces informations, je dois les lui demander et il jugera de mon droit à les avoir. [...] Ce n'est pas normal de ne pas passer par les autorités et d'aller directement dans les zones résidentielles. " (Carnet de terrain, 28.11.2011)

Lors d'une réunion entre fonctionnaires locaux, dont la police communale et les représentants de quartier, un représentant aura évoqué mon enquête. Ne connaissant pas les détails de mes agissements, le policier a dû se sentir remis en cause dans sa capacité de contrôle. La convocation suivante est restée courtoise mais le résultat a été immédiat. De nombreuses rencontres ont soudainement été annulées: "J'ai entendu dire 
par d'autres représentants des unions ${ }^{7}$ que vos questions n'étaient pas agréables donc je ne veux pas vous rencontrer ", répondit un fonctionnaire au téléphone. Un représentant de quartier, jusque-là accueillant et conciliant, vint également me dire qu'il avait eu des ennuis et qu'il lui était désormais interdit de me faire participer à quelque événement que ce soit. La sensation de "terrain facile " que j'avais eue jusque-là correspondait au temps nécessaire pour faire remonter les informations depuis les derniers échelons jusqu'aux autorités. Ma présence devenait envahissante, le réseau de mes contacts s'est alors grippé, comme un mécanisme de fermeture à retardement.

\section{Énoncer, dénoncer, déformer}

Si le contrôle gouvernemental s'exerce sur le chercheur durant son enquête, il s'exerce aussi sur les Vietnamiens et en premier lieu sur leur discours. Dans un contexte où le discours officiel construit la société idéale, la mise à mal du consensus est dangereuse pour la reproduction du régime. Avant même la critique ouverte des autorités, l'énonciation des problèmes, une "parole créatrice, qui fait exister ce qu'elle énonce ", fait émerger des lignes de fractures dans un monde social fantasmé (Bourdieu, $1982: 21)$.

La forme classique du contrôle latent exercé sur la parole est la neutralisation du discours: afin d'éviter d'énoncer des problèmes liés aux ordures, notamment devant un étranger, mes interlocuteurs ont souvent eu recours à l'idiome "cung duoc ", signifiant "ça va ", "rien à signaler ", quand bien même la collecte dysfonctionnait gravement dans leur quartier. Devant une parole plus déliée, le contrôle peut être assuré par un tiers comme lorsqu'un habitué du café où j'enquêtais sermonna mon interlocutrice évoquant la corruption des fonctionnaires: " tu parles trop, on va t'emmener en prison!". Beaucoup de mes entretiens avec des fonctionnaires se sont également déroulés en la présence d'un " chaperon ", issu d'un autre service et mandaté pour rapporter aux supérieurs les propos tenus. Le résultat en est une parole convenue : les fonctionnaires comme certains habitants ont tendance à dresser " un portrait des situations

${ }^{7}$ Organisations paraétatiques de jeunes, de paysans, de femmes, etc. 
comme les choses doivent être plutôt que comme elles sont réellement ${ }^{8}$ (Scott et al., $2006: 33$ ).

Du fait de la prise de risque que suppose le fait même de décrire les problèmes liés à la gestion des déchets, les stratégies de prise de parole sont marquées par des détournements. C'est notamment l'usage de métaphores ou de références au passé national qui permettent de donner de l'aplomb et une légitimité à un discours dénonciateur : "Certains fonctionnaires semblent avoir oublié la camaraderie de la guerre " expliqua un professeur de littérature évoquant la richesse accumulée du président de la province. Certains lieux marqués par l'informalité, comme les cafés, sont par ailleurs propices à la libération de la parole. Ils permettent de jouer sur différentes identités sociales. Au café, M. X n'est plus haut fonctionnaire du ministère de l'environnement risquant la trahison nationale, mais « expert environnemental $»$ indépendant :

Par courriel: "Si vous n'avez pas de lettre de recommandation, nous pouvons nous rencontrer dans un café. [...] Mais je vous rencontrerai en tant qu'expert environnemental. »

Au café : "[...] Ici, nous sommes dans les divisions de gestion, le gouvernement. Peut-être que si vous aviez demandé à rencontrer quelqu'un de ces divisions-là (il montre la colonne de droite sur l'organigramme du ministère), cela aurait été plus facile. C'est parce que ce sont des centres de recherche. Ils sont beaucoup plus accessibles. " (II rit)

\section{[...] " Est-ce alors un problème que nous nous rencontrions dans un café sans cette lettre? "}

"Non, non. C'est la même chose. C'est juste que pour rentrer dans les bureaux il aurait fallu que je demande la permission à mon supérieur. Et puis la salle de réunion était prise cet après-midi. [...] J'aurais demandé la permission, réservé la salle de réunion et ensuite, j'aurais dû faire un rapport à mon supérieur sur ce dont nous aurions parlé. II m'aurait demandé des détails sur la discussion. Mais ce que je dis maintenant, c'est la même chose que ce que j'aurais dit au bureau. C'est juste plus facile. [...] " (Carnet de terrain, 11.11.2011)

\footnotetext{
${ }^{8}$ Ma traduction.
} 
Quoi qu'il en dise, l'entretien avec $M . X$ aurait été différent dans la salle de réunion, avec la présence éventuelle d'un chaperon. Ce que lui juge être un contenu informatif (sur la législation environnementale par exemple) peut être identique au café et au bureau. Mais c'est dans ses détours de langage et ses plaisanteries a priori anodines que l'on peut découvrir de nouvelles informations sur l'organisation institutionnelle : les chercheurs sont accessibles, les gestionnaires non, et ce probablement parce qu'ils manipulent le budget. C'est aussi à cela que s'évalue la transparence des comptes et de la prise de décision.

Parmi les contraintes qui s'exercent sur la parole des interlocuteurs du chercheur, la présence d'un interprète occupe une place particulière qu'il convient d'analyser plus avant. Au Vietnam, le recours à des assistants de recherche est parfois imposé par les institutions locales, même dans le cas où le chercheur maîtrise suffisamment la langue locale pour conduire son enquête seul (Turner, 2013). Dans mon cas, c'est plutôt la faible maîtrise de la langue qui a motivé le recours à deux interprètes successives. La traduction n'est évidemment pas transparente et le rôle primordial de mes interprètes dans la production de l'information mérite d'être analysé. Leur statut a oscillé entre celui de l'assistante de recherche stimulante, s'improvisant traductrice culturelle, et le rôle d'un inquisiteur infiltré, contrôlant le contenu des entretiens.

Se réappropriant la recherche afin de mener les entretiens, mes interprètes se sont focalisées sur une définition très restrictive de mon étude, autour de questions selon elles "strictement liées " aux déchets et à l'environnement: les pratiques quotidiennes des habitants avec les ordures, leur perception de l'environnement. Quand il s'agissait d'étudier les politiques de gestion, le fonctionnement de l'administration, nous étions déjà " hors cadre ". Or, il est difficile de dissocier la question des déchets des politiques publiques associées et donc des problèmes financiers, institutionnels ou politiques. A l'image d'Anne Doquet en pays Dogon, qui s'est vue " accusée, sous le mode d'une plaisanterie sérieuse, d'arracher stratégiquement les informations au moyen de questions innocentes ", il m'a fallu constamment expliquer l'intérêt de mes questions pour ma recherche (Doquet, 2007 : 213). Durant une réunion de travail avec d'autres étudiantes chargées de retraduire des enregistrements, l'une d'elle manifesta sa désapprobation vis-à-vis de mes méthodes, ne comprenant pas pour- 
quoi j'interviewais des fonctionnaires alors que je travaillais sur des questions environnementales. L' " espionite " et la suspicion envers les étrangers auraient donc contaminé jusqu'aux jeunes étudiantes (Dinh Trong Hieu, 1986). Durant un entretien, ma première interprète refusa également de traduire les paroles d'un de nos interlocuteurs qui évoquait des problèmes de corruption et de mauvaise gestion publique, prétextant que cela n'avait rien à voir avec la question des déchets. Elle le fît a posteriori du fait de mon insistance et de notre complicité naissante, ce qui aboutit finalement à des discussions très riches au sujet des carrières de fonctionnaires ou encore de la corruption des policiers. Durant un entretien au café, ma seconde interprète s'est vue faire la leçon par la présidente de I'Union des Femmes de la première commune, accompagnée d'un collègue du département de la culture. Tout en lui demandant de ne pas traduire ses propos, elle lui a expliqué que le rôle d'une jeune Vietnamienne était de montrer "les belles choses " de son pays aux étrangers et qu'au lieu de m'accompagner sur des sites de décharge d'ordures, elle devait me montrer le musée et les chutes d'eau, les deux grands lieux touristiques des environs. En discutant de cet entretien laborieux par la suite, mon interprète me confirma que ces propos reflétaient en substance les consignes générales données aux jeunes Vietnamiens à l'école. Elle m'a affirmé avoir trouvé cette femme tout à fait désagréable et la discussion qui s'ensuivit me permit de connaître sa perception critique des rapports avec les fonctionnaires au quotidien.

A l'issue de cette analyse de l'enquête conventionnelle, on voit comment les errances du chercheur dans son accès à l'information renseignent sur le fonctionnement des institutions et sur les représentations du monde social des différents acteurs. Si le contexte est a priori consensuel, après examen, des lignes de fractures émergent et font entrevoir la latitude des acteurs face au système bureaucratique et leur pouvoir (certes relatif) de contestation.

\section{Écouter et se taire : l'enquête au sein d'un projet bilatéral}

Au vu des lenteurs de l'enquête conventionnelle, j'ai cherché à participer aux activités du projet qui m'étaient accessibles, afin de développer ma connaissance sur la gestion des déchets et mon réseau d'interlocuteurs. Je 
me suis ainsi insérée dans l'institution bilatérale, partageant le quotidien des employés, développant une forme d'intimité avec certains des membres du projet et cultivant un " état de veille ethnographique " (Bouju, 2007 : 141). C'est par ce point de vue, au départ assez extérieur puis finalement impliqué, que j'ai pu comprendre le fonctionnement de cette institution, notamment à travers les usages stratégiques de l'information.

\section{Une alliance institutionnelle conflictuelle}

Le projet repose sur une alliance bilatérale entre le gouvernement vietnamien, représenté par la Division de Planification et d'Investissement du Comité Populaire de la province, et un gouvernement européen, représenté par l'organisme de coopération. Ces deux organismes gouvernementaux, résilients de par leurs traditions institutionnelles sédimentées, se rencontrent dans une petite équipe coordonnant des activités avec divers partenaires locaux. Le projet vise essentiellement à développer des infrastructures, mais une experte et deux assistantes travaillent également sur la " conscientisation " et le " développement des capacités " des acteurs locaux (le "soft component ${ }^{9}$ ). Dans cette cellule de pilotage, seuls le directeur du projet, un ingénieur d'environ soixante ans, et les deux assistantes, âgées de vingt-cinq à trente ans, sont des européens expatriés.

Dans un premier temps, il est nécessaire de décrire l'administration provinciale qui accueille les locaux de la cellule de pilotage du projet afin d'en comprendre la mécanique institutionnelle. Au sein de la Division de Planification et d'Investissement (DPI), les rapports de pouvoir hiérarchiques sont prégnants, comme en témoigne cette description d'une réception organisée dans la cour centrale suite à un tournoi de badminton, le jour de la fête d'indépendance du Vietnam :

[...] Plusieurs tables rondes sont installées au milieu de la cour. Je me retrouve avec le directeur, sa compagne, une assistante, ainsi qu'une collègue d'un autre service et la traductrice du directeur, à la

\footnotetext{
${ }^{9}$ Selon les termes du projet : " awareness raising » et " capacity building », c'est-à-dire des activités "indirectes», non matérielles, accompagnant la construction d'infrastructures. Cette dernière représente à elle seule un budget de plus de $90 \%$ du total.
} 
table des deux grands "patrons " : le président du projet et le président de la DPI. [...] Le plus haut placé est manchot ${ }^{10}$.

Le directeur m'explique les rudiments des règles pour le repas. [...] II a l'air de rechigner à participer à cette réception, mais c'est une obligation [...]. Le principe est que l'on mange et que l'on boit ensemble, tout en respectant la hiérarchie. Aux autres tables, on trinque souvent: "Môt hai ba, Yo !11 " Et tous enfilent leur verre d'une traite [...]. Etant placés à la table des "patrons", nous recevons au compte-goutte la visite des convives des autres tables. Ils viennent trinquer par dessous avec les "patrons " et trinquent d'égal à égal avec le reste de la tablée. Le directeur me conseille judicieusement de ne pas boire trop au début. Chaque visiteur se doit de finir son verre d'une traite et de le montrer au "patron " qui fait mine de s'en fiche : il regarde d'un œil distrait. [...]

Parmi les convives qui viennent saluer leurs supérieurs, une jeune femme s'approche avec un verre de bière plein. Visiblement intimidée, elle trinque avec les deux "patrons ", boit son verre de bière d'une traite après avoir fait de gros yeux et reprit son souffle. C'est un peu laborieux. On sent bien qu'il s'agit d'une obligation. Tout en souriant timidement, elle montre ensuite à un des supérieurs qu'elle a bien fini son verre. II lui fait un geste voulant probablement dire "d'accord, c'est bon " assez nonchalamment. Elle attend l'aval de l'autre supérieur, qui ne s'intéresse pas à elle. Il lui faut visiblement attendre le contrôle pour pouvoir quitter la table [...]. Finalement, le second "patron " lui fait signe et elle s'en va.

Une autre scène marquante est celle de l'assistante étrangère, sollicitée par le supérieur manchot pour lui décortiquer un quartier de pomélo en fin de repas. Après lui avoir fait la cour en lui parlant de mariage et de grande maison [...], ce qui est apparemment habituel, il s'adonne à un jeu qui n'est pas du tout du goût du directeur. Celuici regarde la scène avec dégoût en me disant " oh, c'est tellement bizarre... ". En effet, le manchot veut que l'assistante lui donne la béquée : elle doit porter le quartier de pomélo dépiauté à sa bouche, ce qu'elle rechigne à faire. II insiste. Elle rit, gênée, mais se résout à porter le quartier de pomélo à la bouche du manchot, un brin dé-

\footnotetext{
${ }^{10}$ Le directeur m'a affirmé qu'une infirmité de guerre peut être valorisante pour obtenir un poste de haut fonctionnaire.

11 « Un, deux, trois, Yo!»
} 
goûtée. Tous les convives observent la scène entre curiosité, rire et gêne. La pluie tombe soudainement et le directeur dit à sa compagne : " je crois que c'est le bon moment pour que cela se termine ». Ils partent en catimini. [...] " (Carnet de terrain, 31.08.2011)

La scène est assez représentative des autres repas de travail avec les "patrons » auxquels j'ai pu assister et elle est symptomatique du fonctionnement de la DPI. Le rôle central du président de la DPI et du président du projet (désignés par le terme de " patrons ») et leur liberté d'action en font des personnages à la fois respectés, craints et parfois raillés (certaines de leurs pratiques étant à la limite de la décence). Une des assistantes du projet, qui est incommodée par la bière, s'oblige d'ailleurs à en consommer dans les repas officiels alors qu'elle ne boit que de l'eau dans les repas amicaux. Il s'agit bien de respecter la norme du service administratif. La mise en scène d'un repas officiel illustre par de nombreux aspects la hiérarchie institutionnelle tout comme les rapports de genre institués.

Si la hiérarchie est stricte dans l'administration vietnamienne, au sein de la cellule de pilotage, les rapports de forces sont bouleversés par les pratiques de contournements de différents acteurs. Tout d'abord, deux hiérarchies parallèles existent dans le projet : d'un côté le président de la DPI, le président du projet, le vice-directeur du projet, le comptable, sa secrétaire et les ingénieurs techniques sont tous des fonctionnaires vietnamiens; de l'autre, le directeur étranger, sa traductrice, l'experte vietnamienne ${ }^{12}$ et les deux assistantes sont employés par le projet et donc payés par la coopération. Outre une juxtaposition de grilles de salaire très hétérogènes provocant des jalousies, les autorités devant qui ces employés sont responsables sont différentes. Aussi, les relations de travail franchissant cette frontière interne sont-elles en général conflictuelles.

Le statut des assistantes est plus que représentatif du bouleversement hiérarchique. Dernier maillon de la chaîne sur l'organigramme théorique de l'institution, elles sont mieux rémunérées que l'experte pour qui elles travaillent et outrepassent facilement les échelons hiérarchiques pour

\footnotetext{
${ }^{12}$ Elle a travaillé en Europe pendant une dizaine d'années et est de retour au Vietnam depuis quelques mois. Divorcée, vivant seule avec ses enfants, elle est déterminée dans son travail et visiblement insoumise à la hiérarchie des " patrons ».
} 
défendre leurs points de vue. Elles sont toutes deux en conflits avec le vice-directeur. Subordonné au président du projet et pressé par les injonctions du directeur expatrié, cet ingénieur vietnamien use abondamment de son pouvoir d'obstruction, probablement afin de s'affirmer dans I'institution. Les assistantes cherchent donc souvent à le court-circuiter pour accélérer les procédures. Suite à la proposition d'une assistante avec laquelle il était en désaccord, le vice-directeur la renvoya un jour à son statut subalterne : "Vous n'êtes pas en position de poser cette question ". Formel sur son refus, il lui proposa de soutenir sa proposition au président, son supérieur direct. Provoquée, l'assistante le fit et obtint gain de cause. Depuis, les assistantes et le vice-directeur, bien qu'amicaux dans les couloirs, s'opposent presque systématiquement dans les réunions.

C'est ce même vice-directeur qui devait me délivrer une lettre de recommandation en plaidant mon dossier auprès du président du projet. Or il me semble que j'ai été assimilée aux deux assistantes du fait de nos habitudes communes, de notre ressemblance physique et de ma complicité avec l'une d'elle (qui m'hébergeait gracieusement lors de mes séjours à la capitale provinciale). J'ai donc été " encliquée » (Olivier de Sardan, 2008) et, dans la géographie institutionnelle, je pourrais situer mon point d'ancrage aisément. Outre mon statut d'enquêtrice suspecte, il me semble alors que certains conflits institués se sont quasiment mécaniquement reportés sur moi, gênant notamment mes démarches administratives.

\section{Pratiques informationnelles et tabous de l'institution}

Pour autant, cette réalité conflictuelle ne peut pas être le seul principe organisationnel à l'œuvre, car la reproduction de l'institution dans le temps serait dans ce cas menacée d'éclatement. II devient alors nécessaire de comprendre le projet de développement comme une institution combinant un principe de conflit et un principe de stabilité. Cela se traduit dans les pratiques quotidiennes des acteurs qui, comme dans toute institution, "veulent que la communauté survive sans pour autant abandonner leur autonomie individuelle " (Douglas, 2004 : 75). Un aspect de ce principe de stabilité institutionnelle, ainsi que j'ai pu l'observer, se trouve dans l'usage stratégique de l'information et de la censure par les membres du projet. 
Malgré certains conflits provinciaux, le directeur se doit d'être " transparent " dans sa gestion locale du projet en informant le siège de la coopération installé à Hanoï. II s'autorise cependant à ne pas évoquer des dissensions internes à l'équipe lorsqu'il considère que le siège ne doit pas en avoir vent. Avant une réunion à Hanoï, une assistante demande si la question actuellement en débat d'un voyage d'étude pour les fonctionnaires locaux sera évoquée :

"Non, non, dit le directeur, pas un mot du voyage d'études à Hanoï. C'est quelque chose entre nous et la province! Pas besoin que le siège soit au courant ».

Le directeur du projet a jugé bon de taire la question soulevée et de réserver sa résolution à une discussion interne à l'équipe provinciale. $A$ l'inverse, lorsqu'il juge que le soutien du siège peut être bénéfique à la résolution d'une question locale, le directeur fait remonter des informations à ses supérieurs. C'est le cas de la question des sites de décharge qui engendre des conflits entre communes. La province veut attribuer un site de décharge à chaque district pour contenter tout le monde. Le directeur, convaincu que c'est une erreur, aimerait que le siège de la coopération fasse pression, par le biais de l'ambassadeur lui-même et par répercussion sur toute la pyramide administrative vietnamienne, pour influencer la décision provinciale. Cependant, à Hanoï, on lui a dit " pas de vagues ". Le choix de l'interlocuteur et du circuit de l'information est pensé en amont pour améliorer l'efficacité de l'action. Tout n'est pourtant pas bon à dire et tout interlocuteur ne doit pas être contacté, comme en témoigne l'injonction au silence du siège.

De manière générale, l'information est une ressource pour défendre des intérêts personnels ou les intérêts d'une faction. Au siège national de la coopération, une fonctionnaire vietnamienne, chargée de la supervision du projet provincial, s'immisce dans les affaires locales en voulant imposer le recrutement d'un expert qui n'a pas satisfait les membres du projet sur le papier. Jouant des divisions internes pour imposer son choix, elle argue que les Vietnamiens ont été spoliés dans la procédure et qu'ils n'auraient pas été informés par les expatriés du choix final. Jugeant que cette femme a détruit un lien de confiance ténu entre les deux parties du projet, la réaction du directeur est sans appel : 
"Elle travaille contre nous! Elle ne travaille pas pour les intérêts de la coopération mais pour les intérêts vietnamiens. Et cette fois-ci, je vais me venger. [...] Je peux la faire plonger. J'ai deux dossiers sur elle que je n'ai pas encore utilisés. II y a beaucoup de choses à dire. $Y$ compris sur des affaires de corruption. " (Carnet de terrain, 27.10.2011)

Sous couvert d'une dénonciation reposant sur des valeurs - la lutte contre la corruption -, qui ne sont en réalité mobilisées que de manière stratégique, la principale ligne de fracture dans l'alliance bilatérale en question n'est pas horizontale (entre le local et le siège), mais bien verticale et diplomatique. Ce sont des intérêts nationaux qui sont mis en conflits : « nous » et les Vietnamiens.

Au vu des enjeux internes à la mécanique du projet, la cohésion de l'équipe est en permanence sur la sellette. Cette instabilité institutionnelle est à mon sens compensée par une forme de censure que je qualifie de stratégique : en interne, l'information peut faire l'objet de diverses manipulations mais, vis-à-vis de l'extérieur, il faut témoigner d'une union sans faille, dont l'apparence est indispensable à la pérennité du projet. On peut considérer que la diffusion de l'information répond à une logique de cercles concentriques déterminant le degré de confidence dont les différents acteurs internes, partenaires et externes bénéficient. Les journalistes, partenaires convoités du projet lorsqu'il faut donner de la visibilité aux actions, se changent en indésirables gêneurs dans les cas difficiles, comme lors de la restitution de l'évaluation intermédiaire du projet, faite par une équipe internationale d'évaluateurs indépendants dans un grand hôtel public de la capitale provinciale :

A la pause, le directeur embarrassé arrive auprès du consultant ayant présenté l'évaluation ainsi qu'un de ses collègues avec qui nous discutions l'assistante et moi. "Nous avons un problème. II y a un journaliste dans l'audience. Nous ne savons pas qui l'a invité, comment il est arrivé là. J'imagine que ce n'est pas vous, hein (sourire gêné)? Les Vietnamiens sont très mécontents. Parce que ce n'est pas bon que ce soit public. C'était dur ! » [...]

"Tout de même, vous disiez que nous avons des "résultats pauvres" en renforcement des capacités. Je trouve que c'est très dur. Et puis en ce qui concerne les délais, vous ne prenez pas en compte tous les 
éléments. » Le directeur se lance dans une explication par $A+B$, en montrant ses doigts : " premièrement, [...] deuxièmement, [...] »Ce sont les procédures et les autorisations de la coopération étrangère qui ralentissent. Aussi il termine : "Non, je pense que le projet a fait du bon travail jusque-là, et nous sommes dans les temps. » [...]

Le directeur nie les critiques. Un des consultants est visiblement un peu ennuyé d'être contesté. "On n'est pas à l'ONU! ", blague-t-il. "On est indépendant, on peut émettre des critiques constructives. C'est notre avis. " (Carnet de terrain, 13.12.2011)

Au-delà d'une vision culturaliste des usages de la censure dans la fonction publique vietnamienne, on voit que le directeur expatrié euphémise lui aussi les problèmes ou tait certaines réalités. Les acteurs habituellement en conflit dans l'alliance se retrouvent finalement. L'alliance institutionnelle se maintient parce qu'elle fonctionne aussi sur la base d'un principe de censure stratégique : "Il faut que certaines choses soient continuellement oubliées pour qu'un système cognitif quel qu'il soit puisse fonctionner. » (Douglas, 2004 : 113)

\section{Engagements réciproques}

Dans ce contexte conflictuel, où l'information est une arme précieusement préservée parce que menaçante pour la cohésion institutionnelle, un chercheur peut éventuellement écouter, mais doit surtout se taire. A la suite de ma participation, pourtant autorisée par le directeur, à une importante réunion à Hanoï, j'ai senti un basculement dans mon statut. Touchant de près à des enjeux diplomatiques forts, ma nature allogène s'est soudainement révélée problématique aux yeux du directeur. A la fin de la réunion, il m'a sollicitée en aparté pour me dire, seuls à seuls, que nous devions discuter sérieusement de ma recherche et des bénéfices qu'en retirerait son projet. Par la suite, ma présence fût refusée aux réunions internes de l'équipe. J'ai pu uniquement assister à des activités dans les communes et des réunions " publiques ».

L'implication du directeur dans la définition de ma recherche est allée croissante, mais c'est lorsque ma restitution devant l'équipe du projet approchait, qu'il a frôlé la censure de mon étude. Il m'a convoquée quelques jours à l'avance pour « discuter » du contenu de mon diaporama. 
Comme l'assistante qui m'hébergeait m'avait déconseillée à de multiples reprises d'évoquer des questions institutionnelles (y compris communales), j'ai moi-même largement édulcoré mon propos et j'ai concentré ma présentation sur les pratiques des habitants vis-à-vis des ordures. Le vicedirecteur, manifestant son désintérêt pour mon travail, refusa quant à lui d'assister à ma présentation. Après de longues palabres, il s'est présenté avec plus d'une heure de retard, faisant attendre l'équipe. II a enfin largement commenté mon travail jusqu'à me faire modifier des phrases qu'il n'approuvait pas dans certaines diapositives.

Il faut bien admettre qu'une enquête en situation de développement "transforme un regard auto-critique " en un regard impliqué de "l'anthropologue-acteur [...] parlant du dedans et à la première personne ${ }^{13}$ (Mosse, 2005 : 11). L'acception classique de la recherche sur un objet autonome, comme le développement, laisse place à celle d'une anthropologie impliquée, qui prend en compte la réflexivité du chercheur. Cette implication soulève par ailleurs une grande difficulté à restituer le travail de recherche hors de l'institution, d'autant plus dans le cas d'un matériau empirique non conventionnel. Quel doit être l'engagement du chercheur vis-à-vis de ses interlocuteurs ? L'éthique de l'anthropologue réside à mon sens dans la rigueur ethnographique, pour laquelle plaide Oliver de Sardan (2008), qui permet à la production scientifique d'" être conséquent[e] "(Fassin, 2005). La justesse du propos, au service de la science anthropologique et non tournée vers la personne du chercheur, est une manière de créer cette "symétrie [...] de concernement" et d'implication dans les histoires vécues de nos interlocuteurs (Héraut, 2007 : 105).

\section{Conclusion}

En 1990, Bourdieu faisait le constat suivant :

"Je pense que si la théorie de l'Etat, dans l'état de délabrement où elle se trouve, [...] peut se perpétuer, c'est parce qu'elle se promène dans un univers indépendant de la réalité " (Bourdieu, 2012 : 46).

${ }^{13}$ Ma traduction. 
Cet appel à conjuguer empirie et théorie dans la production du savoir sur l'Etat me paraît fondamental dans le projet qui est le nôtre d'étudier les politiques publiques en contexte de développement. N'est-ce pas en expérimentant les institutions, pour le meilleur et pour le pire, que le chercheur peut comprendre leurs mécanismes de fonctionnement?

Les institutions, "inventions organisationnelles ", visent à transformer le réel sur la base d'un point de vue situé sur le monde social. Aussi, et parce qu' « une fiction sociale n'est pas fictive » (Bourdieu, 2012 : 53), il est intéressant de comprendre les processus de production du discours par les institutions, les mécanismes du consensus, de la censure et d'analyser les usages stratégiques de l'information par les acteurs. Le tropisme réflexif du socio-anthropologue, analysant les conditions pratiques de son accès à l'information, ses difficultés à obtenir des autorisations de recherche, etc., est alors un positionnement heuristique pour analyser un fonctionnement institutionnel. La question de la réflexivité doit donc être vue comme un appel à construire une "épistémologie de terrain " (Olivier De Sardan, 2008), alimentant en permanence la réflexion théorique par l'expérience empirique du chercheur. A la manière du judoka, utilisant la force de son adversaire pour lui infliger une défaite, les contretemps d'une enquête ethnographique en situation de développement peuvent nourrir durablement la réflexion théorique du chercheur sur les contextes d'interventions.

\section{Bibliographie}

BLUNDO G., LE MEUR P.-Y. (eds.), 2009, The Governance of Daily Life in Africa, Ethnographic Explorations of Public and Collective Services, LeidenBoston, BRILL.

BONNIN C., 2011, "Doing Fieldwork and Making Friends in Upland Northern Vietnam : Entanglements of the Professional, Personal and Political ", Conférence 3-5 mai 2011, Montréal, Mc Gill University.

BOUJU J., 2007, "La vie quotidienne comme terrain, ou la valeur heuristique de l'implication de l'ethnographe assistant technique en coopération ", in Leservoisier O., Vidal L. (dir.), L'anthropologie face à ses objets, Nouveaux contextes ethnographiques, Paris, Archives contemporaines : 139-151. 
BOURDIEU P., 2012, Sur l'Etat. Cours au Collège de France 1989-1992, Paris, Raisons d'agir/Seuil.

BOURDIEU P., 1982, Ce que parler veut dire. L'économie des échanges linguistiques, Paris, Fayard.

CONDOMINAS G., 1965, L'exotique est quotidien, Sar Luk, Viet-nam central, Paris, Plon.

DINH TRONG HIEU, 1986, "L'accès au terrain en pays socialistes de I'Indochine, pour les recherches en sciences sociales ", in Gast M., Panoff M. (eds.), L'accès au terrain en pays étranger et outre-mer, Paris, L'Harmattan : 6990.

DOQUET A., 2007, "L'enquête en "situation ethnologique" ou l'exercice nécessaire de la réflexivité ", in Leservoisier O., Vidal L., L'anthropologie face à ses objets, Nouveaux contextes ethnographiques, Paris, Archives contemporaines : 205-222.

DOUGLAS M., 2004 [1986], Comment pensent les institutions ?, Paris, La Découverte.

FASSIN D., 2005, "L'innocence perdue de l'anthropologue: remarques sur les terrains sensibles ", in Bouillon F., Fresia M., Tallio V. (eds.), Terrains sensibles, Expériences actuelles de l'anthropologie, Paris, EHESS, Centre d’Etudes Africaines : 97-103.

HERAULT L., 2007, "Faire de l'anthropologie en "terrain transsexuel" ", in Leservoisier O., Vidal L., L'anthropologie face à ses objets, Nouveaux contextes ethnographiques, Paris, Archives contemporaines : 97-107.

MORIN E., 2005, Introduction à la pensée complexe, Paris, Seuil.

MOSSE D., 2005, Cultivating development : An ethnography of aid policy and practice, New York, Pluto Press.

NØRLUND I., 2007, « Civil society in Vietnam, Social organizations and approaches to new concepts ", ASIEN The German Journal on Contemporary Asia, 105, Hamburg, Deutsche Gesellschaft für Asienkunde e.V. : 68-90.

OLIVIER DE SARDAN J.-P., 2008, La rigueur du qualitatif, Louvain-la-Neuve, Bruylant-Academia.

SCOTT S., MILLER F., LLOYD K., 2006, « Doing Fieldwork in Development Geography, Research Culture and Research Spaces in Vietnam ", Geographical research : 28-40. 
TURNER S. (ed.), 2013, Red stamps and gold stars. Fieldwork dilemmas in upland socialist Asia, University of British Columbia Press, Vancouver.

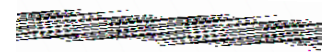

Mikaëla Le Meur est doctorante en anthropologie au Laboratoire d'Anthropologie des Mondes Contemporains,

à l'Université Libre de Bruxelles (Belgique)

E-mail : mlemeur@ulb.ac.be 